\title{
Running laboratory experiments using the RSX operating system and FORTRAN on the PDP-11
}

\author{
STEPHEN E. EDGELL and STANLEY A. HERTEL \\ University of Louisville, Louisville, Kentucky
}

\begin{abstract}
A system of FORTRAN-callable subroutines designed to facilitate running laboratories with the RSX operating system on a PDP-11 computer is presented. The subroutines include ones to give the FORTRAN programmer timing capability, better control of CRT input and output, control of a parallel-bit input and output interface, control of an analog-to-digital interface, and control of a digital-to-analog interface.
\end{abstract}

There are four ways that an experimenter can effect the computer programming necessary to run an experiment. First, the experimenter can program the computer in assembler language. Although this approach allows maximum flexibility and direct control of the various laboratory input/output $(\mathrm{I} / \mathrm{O})$ interfaces, it is quite cumbersome for even a very experienced programmer, and out of the reach of a novice programmer. The second approach is to use a high-level programming language. Although this is much less cumbersome and more accessible than assembler language to novice programmers, it is often difficult, if not impossible, to achieve the required timing and controlling of interfaces. The third alternative is for the experimenter to use a high-level language developed specifically for experiment control. Indeed, several such languages have been developed, for example, PROSS (Scholz, 1972). However, these languages have usually been found to be cumbersome to use, and do not give sufficient error detection. The reason for these difficulties is the large number of hours it takes to develop a highquality compiler such as those found in most implementations of standard higher level languages. No one is willing to invest this large amount of time for a language of limited use. Finally, the experimenter can use a "canned" program designed to facilitiate running experiments. Again, several of these have been developed, for example, MEL (Schneider, 1988). However, these programs were designed to facilitate a particular research paradigm or area. Even within that paradigm, they do not have full flexibility, but rather limit the experimental designs that can be run.

One solution to this dilemma is to develop a set of subroutines that are accessible from a higher level language. Thus, the power and ease of programming in the higher level language is retained, while the subroutines effect the necessary timing and control. In addition, the

Correspondence concerning this article should be sent to Stephen E. Edgell, Department of Psychology, University of Louisville, Louisville, KY 40292. programmer-even a novice-is already familiar with the higher level language. There are many examples of such developments. For example, Edgell (1974) and Castellan (1975) have reported such a system for use in BASIClanguage programs. Restle and Brown (1969), Hendry \& Lennon (1973), and Kaplan (1977) have reported such systems for FORTRAN. The purpose of the present paper is to present another such system. It is designed for the PDP-11 computer using the RSX operating system.

The present subroutine system uses FORTRAN as the higher level language. Previous reports have pointed out that FORTRAN is the best and most popular language for adapting in this manner for laboratory use (Castellan, 1976; Gregg, 1977). The RSX operating system is a multiuser, real-time system. Included in it are many FORTRAN-callable subroutines for implementing process control. However, many of the available subroutines are for obsolete interfaces, or require a level of understanding of process-control programming not usually found in novice programmers. Moreover, there are several functions necessary for controlling laboratories that are not available in the RSX set of FORTRAN-callable subroutines. Thus, we developed a set of subroutines with three goals in mind: (1) they should be easily accessible to novice programmers, (2) they should accomplish all the needed tasks of a psychology laboratory, and (3) they should use currently available interfaces.

For more than a decade, authors have been predicting that the single-user microcomputer would replace the minicomputer in psychology laboratories (Gregg, 1977). It seems that this transition is almost complete now. However, as Kaplan (1981) points out, there is much to recommend the minicomputer over the microcomputer. One advantage of the minicomputer is the sophistication of the operating system. Indeed, the RSX system is excellent for controlling laboratories. Another advantage is that the level of documentation is usually much higher on the minicomputer than on the microcomputer. This documentation is often required for the successful running of laboratories. One of the main factors cited for the 
microcomputer is its lower cost. However, many minicomputers are really microcomputers, and their cost is comparable. The cost can even be favorable to the minicomputer when it is amortized over the number of users that a multiuser system such as RSX allows. We have attempted to retain the power of the multiuser environment in designing the subroutines described below. However, there are also some problems associated with the multiuser environment. A discussion of some of these problems can be found in the user's manual described below, and in Forshee (1974).

\section{Implementation and Hardware}

These subroutines were developed on a PDP-11/23 computer under version 4.1 of the RSX-11M operating system. However, they should run with little or no modification on later versions of the software and on other PDP11 machines. The subroutines will work with any configuration of serial line units. We use one DZQ11 and two DLVJ1-M interfaces. The subroutines will run one DRV 1 1-J 64-bit parallel I/O board. The subroutines will also handle one AXV11-C analog-to-digital and digitalto-analog board. A KWV11-C clock is required to run the AXV11-C board. The subroutines can be easily modified to run more than one DRV11-J and/or AXV11-C interface. All of the subroutines are modular, and one may use any subset as needed. All of the subroutines were written to use standard interface addresses, but these can easily be changed by altering one parameter at the beginning of the program. The names of the subroutines were chosen so that there would be no conflicts with any routines or entry points currently in the system library on RSX. Hence, these subroutines can be installed in the system library in order to facilitate their use. The subroutines are well documented to aid the more advanced user, and a user's manual is available from the authors to aid all users. The following discussion is of a general nature. For more information on using these subroutines, the reader is referred to the user's manual.

\section{The Subroutines}

Sleep. The RSX system has an extensive set of subroutines to handle event flags. However, most novice programmers find the concept of event flags difficult to grasp. Hence, we have simplified this situation with a subroutine called SLEEP. Conceptually, the program performs whatever is required at the current time and then "goes to sleep" by calling this subroutine. SLEEP will then return to the user's program (i.e., the program will "awake") whenever something occurs (e.g., a timer times out or a response is made) that requires the program to service it. Since the return is to the next statement following the call to SLEEP, the top-entry problem discussed by Scholz (1972) is avoided. Most of the subroutines in this package were written to use the SLEEP subroutine. Different users can simultaneously use this subroutine without any special programming, since all the events are local to the particular user's program. Further- more, while a program is "asleep," the computer is free to run other users' programs. Hence, the full power of the multiuser environment is retained.

Timing. One of the most fundamental requirements of running a laboratory is the ability to use timers that allow events to be spaced and the time between two events to be measured accurately. Although RSX provides the standard WAIT subroutine, this approach has well-known shortcomings (Kaplan, 1977). In order to overcome these problems, we used the soft-timer approach (see Scholz, 1972). In our implementation, an array in memory is set up so that each word acts as an automatic timer. Any positive value placed in one of the words is decremented every $\Delta t$ time units until the value reaches zero. Upon reaching zero, the SLEEP subroutine will return, thereby awakening the program. The timer can also be interrogated at any time. Negative values will have no effect and are not changed; thus, they can be used as flag values. A common practice is to use -1 to indicate that the timer is not currently in use. Any number of timers can be set up by one call to TIMRS. This call also sets up a continuously upcounting latency counter as one word in the user's program. By saving the value of this counter and subtracting it from a later value, the number of $\Delta t$ time units that have elasped can be measured. (It should be noted that in base 2 arithmetic, which the PDP-11 uses, there is no wraparound problem with such a counter unless more than 65,536 time units have elapsed.)

The value of $\Delta t$ is also set by the call to TIMRS. This value is a whole number that represents multiples of $1 / 60$ th of a second. By modifying the program to use the KWV11-C clock, a user can achieve more accurate timing, but this is usually not needed. Indeed, our timer is adequate if a CRT is being used in the experiment, because accuracy beyond $1 / 60$ th or $1 / 30$ th of a second, depending upon the CRT, is imaginary (see Lincoln \& Lane, 1980). Each user's program can have its own array of timers and latency counters. Thus, the full power of the multiuser system is retained.

Serial line units. Serial line units are often used to drive CRTs in psychology experiments. However, FORTRAN READs and WRITEs are not handy for controlling serial line units in the experimental environment. We developed a set of subroutines to give the FORTRAN programmer complete control over the input and output to the CRTs. A two-word array for each CRT to be used is set up in the user's program. Any character typed on the associated CRT is immediately and automatically put into the first word of that array. The program will then wake up from SLEEP and can service the character. By resetting the first word to zero, the user's program can tell that the character has been serviced and can go back to SLEEP to wait for another input. The value of the latency counter at the time the character was received by the computer is placed in the second word. Thus, latencies for pressing CRT keys can be easily obtained. This subroutine, TERM1, is called once in the user's program to set this up. The CRT is attached and given some degree of pro- 
tection from other users and from subjects who use the keyboard. For example, a control-C keystroke is passed to the array just like any other character and does not give the subject access to the operating system. The user also has complete control over feedback to the subject, as the character is not even echoed to the CRT. Again, several users can use this subroutine simultaneously (of course, they must be using different serial line units).

Output to a serial line unit is done by the subroutine TOUT1. This subroutine merely starts the output, and the program can go to SLEEP until the output is complete. At that time, the program will awaken and a flag will be set to indicate that the output is finished. Knowing when the output is complete is useful for saving the current value of the latency counter, so that latency timing begins when the output is all on the screen. This subroutine can be called repeatedly to queue up output to the same CRT. Simultaneous output to different CRTs can also be achieved. Any character, including control characters used to access the full capabilities of modern CRTs, can be output with this subroutine. Several users can use this subroutine simultaneously.

There are several subroutines to allow various characteristics to be set under program control for the various serial line units. Most of these characteristics can be set by system commands, but it is often convenient to be able to do such in a program. Subroutine RPA sets the unit to read and pass all, while NORPA sets the unit to its usual state, canceling the read-and-pass-all state. The subroutine NOBRO sets a CRT so that other users cannot broadcast a message to it, and BRO resets it so that such messages are allowed. A terminal can be "slaved" by calling the subroutine SLAVE, and unslaved by calling NOSLAV. A subroutine to "attach" a terminal is also included in case the TERM1 subroutine is not being used. This subroutine is called ATTACH, and to detach the subroutine, DETACH.

Parallel input/output. The DRV11-J has four words of 16 bits each, for a total of 64 bits of input or output. We have dedicated the first two words to input and the second two words to output. The two input words can be fetched by calling a subroutine called DRVIN. Any number of users can use this subroutine, but they must program the computer to ignore the status of input bits not assigned to them. A subroutine called IBIT, which returns the value of a specified bit, has been included to facilitate this. DRVIN was programmed so that changing one equate at the beginning of the program will cause the inputs to be either complemented or not complemented. This is helpful if the hardware connected to the DRV11-J is inverting.

When sending the output to a parallel interface when more than one user is using it (different bits, of course), the user must be careful to make his or her use independent of other users'. In order to do this, the output subroutine DRVOUT has two arrays. Each bit set in one array will set an associated output bit in the DRV11-J. Each bit set in the other array will clear an associated bit in the DRV11-J. Bits not set in either array will be left unchanged in the DRV11-J. Hence, as long as the users are careful to set only bits that are assigned to them in either array, no user will affect any other. A subroutine called BITSET has been included to facilitiate the setting of bits in a word.

Both DRVIN and DRVOUT use a shared common named RTCOM. This common is 32 words long and must be installed in memory. In our system, we have no loadable drivers, and thus were able to use the loadable driver partition that had been reduced to 32 words by VMR during sysgen. If such a ready-made partition is not available, then one must be created and installed. This can be done in the startup command file. When linking any program that uses DRVIN or DRVOUT, the user must specify an option to the task builder (linker) that the program is to use the common RTCOM. By putting the symbol table from RTCOM in LB: 1,2 the option COMMON is used. If the symbol table is in the user's account, then the option RESCOM is used (refer to the task builder manual in RSX and the user's manual for more details). Both DRVIN and DRVOUT also use the task DRV. A separate task, DRV, is mapped to the I/O page in the PDP-11 so that control of the DRV11-J can be effected without constraining the size of the user's program. DRV must be installed and executed during system startup. It remains suspended until needed by DRVIN or DRVOUT.

One problem with DRVIN is that a bit changing in the DRV11-J will not awaken the program from SLEEP. There are two subroutines that solve this problem. Unfortunately, only one of them can be used at any one time, and by only one user. The first, DRVI1, makes the first eight bits of the first register of the DRV11-J awaken the program from SLEEP whenever they change from low to high or from high to low. Only one of these transitions can be implemented, and the transition is selected by changing one equate at the beginning of the program. This subroutine also sets up a two-word array in the user's program in which the value of the first two words is placed whenever one of the first eight bits changes. This subroutine is called only once in the program to set up this action. The other subroutine, DRVI2, works the same way, except that all 16 bits of the first register work in this fashion. These subroutines do not require the common described above. However, since they connect to interrupt a user's program, using one of them must be linked (built) with level 0 privilege and the system-symbol table must be included (refer to the user's manual for more details).

Analog to digital. The subroutine ATOD1 is used to take a number of samples from the analog-to-digital interface, which is part of the AXV11-C. The user specifies the number of samples, the channel, and the frequency. After calling ATOD1, the user calls SLEEP, which will awaken when the sampling is done. An error flag must be checked both after the call and after awakening from SLEEP to be sure that the data is accurate. The KWV11-C clock card is hardwired to the AXV11-C I/O board in order to insure absolute accuracy of the sam- 
pling rate. The samples are picked up under interrupt control and put into one of two 50-word alternating buffers. These buffers are flushed to the user's array under an asynchronous system trap. All of this is transparent to the user. However, if the user's program is not of sufficient priority and the sampling rate is high, then the flush will not be fast enough. This will be evidenced by the error flag upon return, so the user will know that the data are not good. This buffering method is required because of the special mapping scheme used by RSX under interrupt servicing. Also, interrupts from other devices, such as terminals, will delay servicing the interrupt, and possibly cause a sample to be missed. Again, this will be noted by the error flag upon return from SLEEP. This problem is due to a hardware design error on the part of DEC. The AXV11-C interrupts at the lowest priority hardware level. There is no solution for this problem except to avoid other interrupts or to sample at a lower rate. Since ATOD1 connects to interrupt, the statements about privilege and the system-symbol table mentioned for DRVIl above also apply here. Only one user at a time may use this subroutine.

Digital to analog. Analog voltages can be output to the AXV11-C digital-to-analog ports by using the subroutine DTOA. This subroutine also uses the common RTCOM and the task DRV. Hence, the comments made about these above also apply here. Only one user at a time may use this subroutine.

\section{Availability and License}

A source listing of this system and the user's manual are available at no cost from the authors. Persons who desire the source files on an IBM-compatible floppy disk should send a blank disk in a reusable mailing container to the authors. The source files can also be sent via
BITNET. The authors give full permission for any nonprofit use of this system, including copying, provided that full credit is given to the authors. Any for-profit use is prohibited.

\section{REFERENCES}

Castellan, N. J., JR. (1975). The modern minicomputer in laboratory automation. American Psychologist, 30, 205-211.

Castellan, N. J., JR. (1976). Standards: Real-time extensions to programming systems. Behavior Research Methods \& Instrumentation, 8, 207-210.

EDGELl, S. E. (1974). Real time EXF for single user basic [Computer program]. Decus Program Library No. 11-167.

ForsheE, J. C. (1974). Design and organization in a computerized psychology laboratory. Behavior Research Methods \& Instrumentation, 6, 146-148.

GrEGG, L. W. (1977). Maximizing the mini-uses of on-line computers. Behavior Research Methods \& Instrumentation, 9, 67-71.

HENDRY, D. P., \& LENNON, W. J. (1973). A FORTRAN based control and data analysis system for behavioral experiments. In B. Weiss (Ed.), Digital computers in the behavioral laboratory (pp. 141-190). New York: Appleton-Century-Crofts.

KAPLAN, H. L. (1977). Clock-driven FORTRAN task scheduling in a multiprogramming environment. Behavior Research Methods \& Instrumentation, 9, 176-183.

KaPLaN, H. L. (1981). Traditional minicomputers: A continuing role in the laboratory. Behavior Research Methods \& Instrumentation, 13, 213-215.

Lincoln, C. E., LANe, D. M. (1980). Reaction time measurement errors resulting from the use of CRT displays. Behavior Research Methods \& Instrumentation, 12, 55-57.

Restle, F., \& Brown, T. V. (1969). A computer running several psychological laboratories. Behavior Research Methods \& Instrumentation, 1, 312-317.

SCHNEIDER, W. (1988). Micro Experimental Laboratory: An integrated system for IBM PC compatibles. Behavior Research Methods, Instruments, \& Computers, 20, 206-217.

Scholz, K. W. (1972). Computerized process control in behavioral science research. Behavior Research Methods \& Instrumentation, 4, 203-208. 